UVX 2010 (2011) 83-89

DOI: $10.1051 / \mathrm{uvx} / 2011012$

(C) Owned by the authors, published by EDP Sciences, 2011

\title{
Inner-shell photo-ionisation x-ray lasing
}

\author{
S. Jacquemot ${ }^{1,2}$, M. Ribière ${ }^{3}$, A. Rousse ${ }^{4}$, S. Sebban ${ }^{4}$ and K. Ta Phuoc ${ }^{4}$ \\ ${ }^{1}$ LULI, École Polytechnique, CNRS, CEA, UPMC, 91128 Palaiseau, France \\ ${ }^{2}$ CEA, DAM-DIF, 91297 Arpajon, France \\ ${ }^{3}$ CEA, DAM-Gramat, 46500 Gramat, France \\ ${ }^{4}$ LOA, ENSTA Paris Tech, École Polytechnique, CNRS, Chemin de la Hunière, \\ 91761 Palaiseau, France
}

\begin{abstract}
In this paper, we study the possibility to produce femtosecond monochromatic coherent (" $\mathrm{x}$-ray laser") pulses in the $\mathrm{keV}$ range from inner-shell photo-ionisation of a gaseous medium by a laser-driven betatron pump source. A collisional-radiative model and a radiative transfer post-processor have been built to determine the lasing plasma kinetics, assess the local gain coefficient along the $\mathrm{K} \alpha$ transition in the ASE regime, and finally compute the x-ray laser intensity at saturation, to infer the key parameters for an experimental demonstration.
\end{abstract}

\section{INTRODUCTION}

Realizing a table-top ultra-short monochromatic coherent radiation source in the X-ray range is of real interest for many applications as, for instance, sample analysis at the nm scale using holography or diffraction pump-probe experiments with very high temporal resolution. Various schemes based on laser-plasma interaction have been proposed but, while high-order harmonic generation and optical field ionisation x-ray lasing are currently limited, in terms of wavelength, to a few hundreds of Ångströms, only inner-shell photo-ionisation (ISPI) X-ray lasing, first proposed by Duguay and Rentzepis [1], allows going down to the $\mathrm{nm}$, which makes it especially attractive. As shown on the partial energy diagram below (figure 1), it rests on the production of K-shell vacancies (labelled [b1s]) by an external x-ray pump source. To induce $\mathrm{X}$-ray lasing along the [1s]-[2p] $\mathrm{K} \alpha$ line, this source has to be intense enough, at least at energies just above the K-shell ionisation threshold $\left(\mathrm{E}_{\mathrm{th}}\right)$, and short enough, to overcome fast depopulation through Auger decay.

Numerous extensive studies have been conducted since the 70's to estimate the required pump power density for significant population inversion [2]. Atomic kinetic modeling has for instance shown that significant gain coefficients can be achieved in neon at $1.5 \mathrm{~nm}$ for a blackbody pump source with a maximum temperature ( $\left.\mathrm{T}_{\mathrm{m}}\right)$ close to $1 \mathrm{keV}$ and a duration below 50 fs (figure 2).

To produce such a source, the first proposed experimental design consists in focusing - along a line - an adequate $(1-10 \mathrm{~J}, \sim 50 \mathrm{fs})$ driving laser pulse on a high-Z material, but it suffers from difficulties linked to the necessary implementation of a sophisticated travelling wave system (related to the very short expected duration of the population inversion: $\sim 20 \mathrm{fs}$, i.e. $\sim 6 \mu \mathrm{m}$ at the speed of light) [3] which prevents from demonstrating the lasing scheme. In order to overcome these problems, the possibility of using betatron radiation, as produced by a laser-driven relativistic electron beam, and longitudinal pumping have been recently proposed (figure 3) [4,5].

This is an Open Access article distributed under the terms of the Creative Commons Attribution-Noncommercial License 3.0, which permits unrestricted use, distribution, and reproduction in any noncommercial medium, provided the original work is properly cited. 


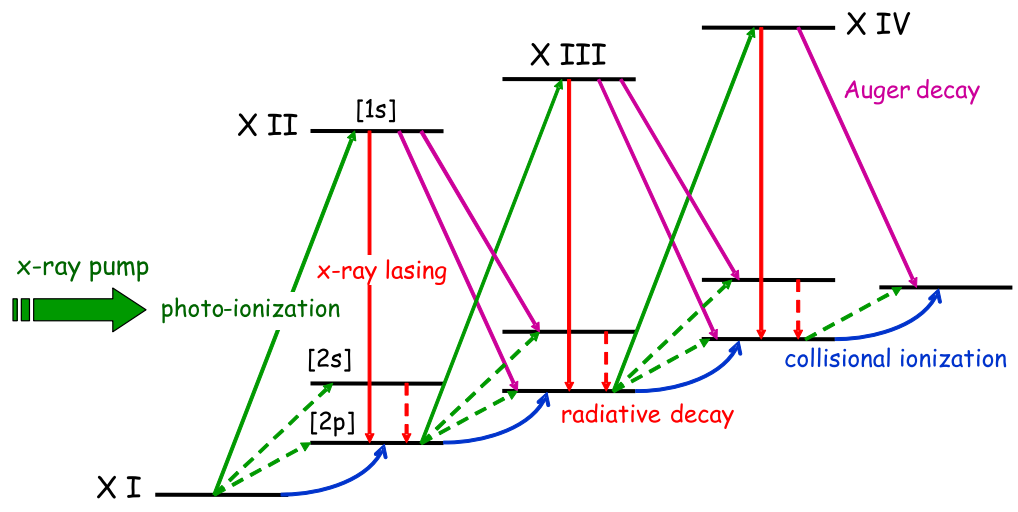

Figure 1. Partial energy diagram of an ISPI x-ray laser (X I, X II, ... referring to the neutral, singly ionised, ... atom and $[\mathrm{nl}]$ being used to label shell vacancies: $\mathrm{K}=1 \mathrm{~s}, \ldots)$.

blackbody $T_{m}=1200 \mathrm{eV}$ - Be filter $3.5 \mu \mathrm{m}$

pure $\mathrm{Ne}: \mathrm{n}_{\mathrm{i}}=10^{20} / \mathrm{cm}^{3}, \mathrm{Te}_{0}=0.025 \mathrm{eV}$

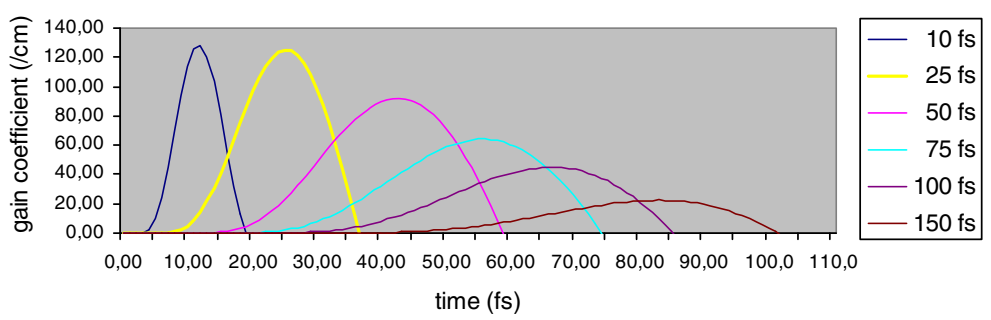

Figure 2. Temporal evolution of the local gain coefficient (assuming a neon lasing medium at ambient temperature and with a density $\mathrm{n}_{i}$ equal to $10^{20} / \mathrm{cm}^{3}$ ) pumped by a blackbody radiation source at $\mathrm{T}_{\mathrm{m}}=1.2 \mathrm{keV}$ (the low-energy component of its spectrum being filtered with the help of a $3.5 \mu \mathrm{m}$-thick Be foil) and of duration ranging from 10 to $150 \mathrm{fs}$

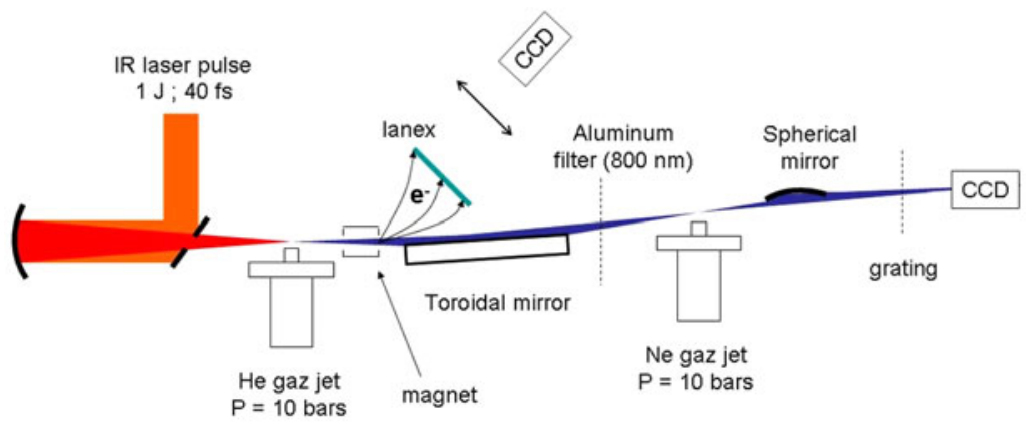

Figure 3. Experimental set-up for ISPI x-ray lasing in neon $(1.5 \mathrm{~nm})$.

\section{DESCRIPTION OF THE BETATRON PUMP SOURCE}

When an intense laser pulse is focused into a low- $\mathrm{Z}$ jet, the ponderomotive force expels the electrons out of the strong laser field region, thus creating an ion cavity backwards. Because of the fields resulting from charge separation, electrons trapped in this 'bubble' are longitudinally accelerated up to 


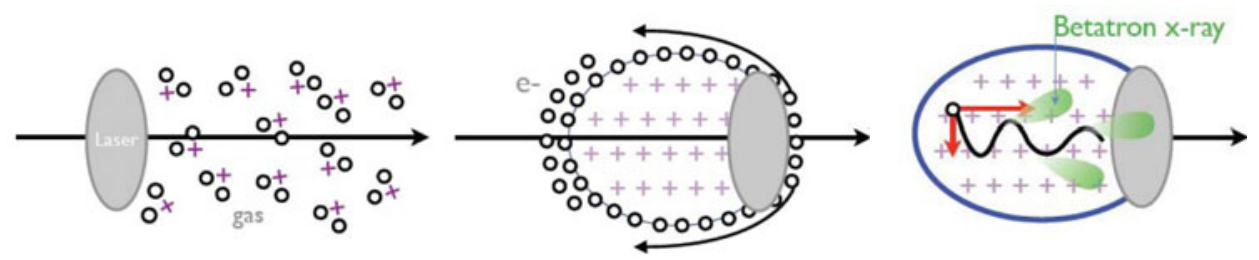

Figure 4. Schematic principle of a $\mathrm{keV}$ betatron source.

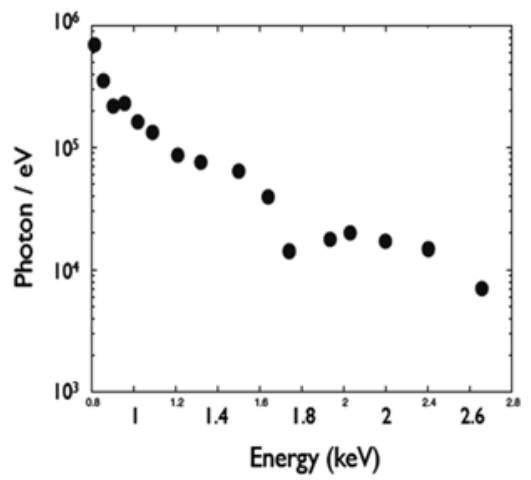

Figure 5. Experimental betatron spectrum [6].

relativistic energies and, if off axis, undergo oscillations, inducing radiation emission with a broadband synchrotron-like energy spectrum (hence the betatron - or $\beta$ tron - appellation) (figure 4). This process has been widely studied at LOA [6]. Using a $30 \mathrm{TW}$ laser focused onto a helium gas jet (at density close to $10^{19} / \mathrm{cm}^{3}$ ), A. Rousse and co-workers succeeded in accelerating electrons up to $200 \mathrm{MeV}$ and producing a rather collimated $(\sim 20 \mathrm{mrad}$ ) and brief (estimated to be less than $100 \mathrm{fs}) \beta$ tron source with more than $10^{8}$ photons above $\mathrm{E}_{\mathrm{th}}(405 \mathrm{eV}$ for nitrogen and $870 \mathrm{eV}$ for neon: figure 5$)$. Assuming a source size close to $4 \mu \mathrm{m}^{2}$ and a duration ( $\mathrm{t}_{\mathrm{p}}$ ) of $10 \mathrm{fs}$, $\mathrm{x}$-ray pump intensities above $10^{13} \mathrm{~W} / \mathrm{cm}^{2}$ could then be reached. These numbers will be used as input parameters for the models described in the following sections, assuming furthermore filtering of the energy spectrum below $\mathrm{E}_{\mathrm{th}}$ and a dependence on time of the $\beta$ tron source power density given by $\sin ^{4}\left(\omega \mathrm{t} / \mathrm{t}_{\mathrm{p}}\right)$.

\section{ISPI X-RAY LASER ATOMIC KINETICS}

A collisional-radiative model has then been built to describe the temporal evolution of the involved level populations (i.e. the ground states $1 \mathrm{~s}^{2} 2 \mathrm{~s}^{2} 2 \mathrm{p}^{\mathrm{n}}$ and the "hollow" $1 \mathrm{~s} 2 \mathrm{~s}^{2} 2 \mathrm{p}^{\mathrm{m}}$ and $1 \mathrm{~s}^{2} 2 \mathrm{~s} 2 \mathrm{p}^{\mathrm{m}}$ configurations of the first ionisation stages: figure 1) and thus the local gain coefficient along the K $\alpha$ line. The free electron energy distribution function is self-consistently calculated and all the populating/depopulating processes are included. Recombinations and electron-electron relaxation are however neglected, as they both occur on a longer timescale than the expected population inversion. Using as inputs the abovedescribed experimental parameters, the only "free" parameters of the simulations are the duration of the $\beta$ tron emission and the initial density of the lasing medium $\left(\mathrm{n}_{\mathrm{i}}\right)$.

The influence of these two quantities on the local gain coefficient $\alpha$ is presented below.

A strong influence of the pump duration is exhibited (the shorter, the better) which demonstrates the benefit, for the ISPI lasing scheme, from the ultra-short nature of the $\beta$ tron source. The density has to be kept sufficiently low to prevent collisional ionisation of the $\mathrm{L}$ shell to destroy population 

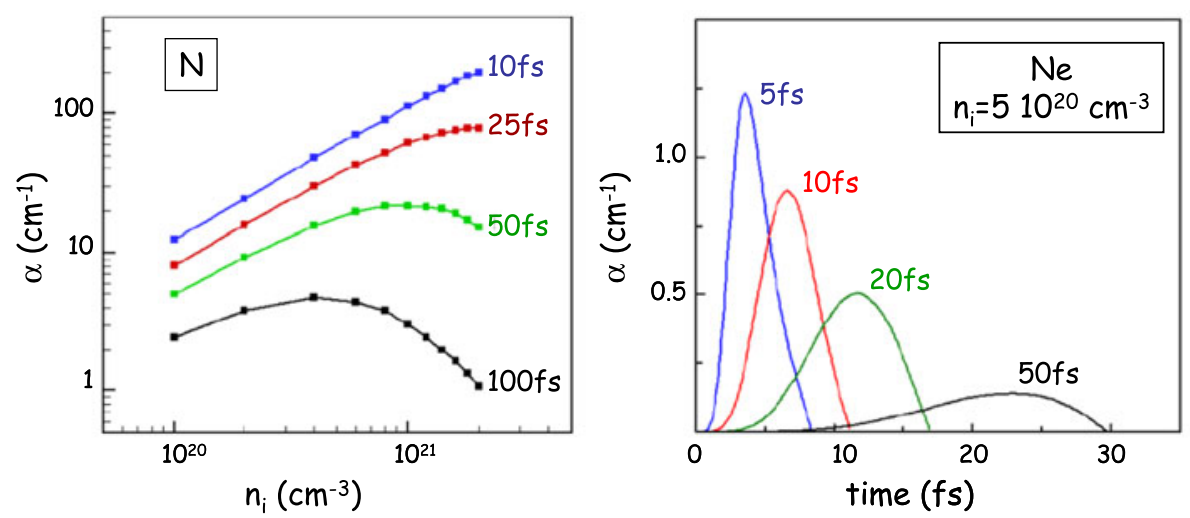

Figure 6. (left) Evolution, with respect to the initial gas density $\mathrm{n}_{\mathrm{i}}$ and to the $\beta$ tron pump duration, of the local gain coefficient obtained at $3.1 \mathrm{~nm}$ in a nitrogen medium. (right) Temporal evolution of the local gain coefficient obtained at $1.5 \mathrm{~nm}$ in a neon medium ( $\mathrm{n}_{\mathrm{i}}$ being fixed at $510^{20} / \mathrm{cm}^{3}$ ) for various $\beta$ tron pump durations.

inversion (figure 6, left), its optimum value depending on the pump duration: the shorter, the higher. All the calculations presented hereafter have been performed with an 'average' density of $510^{20} / \mathrm{cm}^{3}$. The lower gain values obtained in neon (more than one order of magnitude: figure 6, right) can be explained by the shorter lifetime of the inner-shell vacancies (the Auger lifetime varying from $7 \mathrm{fs}$ for nitrogen to $2.75 \mathrm{fs}$ for neon) and the higher K-shell threshold energy, thus leading to a smaller available pump photon density (for the given experimental $\beta$ tron source). It appears then that the currently available experimental $\beta$ tron source could probably induce lasing in nitrogen at $3.1 \mathrm{~nm}$ but that improvements have to be achieved to go to shorter wavelengths.

\section{NUMERICAL OPTIMISATION OF THE BETATRON PUMP SOURCE}

Monte-Carlo 2D simulations have been performed to evaluate the $\beta$ tron radiation produced by more energetic electron beams as those recently demonstrated on a 200TW-class laser [7] or as given by scaling laws [8]. Four cases have been investigated: (a) $\mathrm{P}_{\text {las }}=200 \mathrm{TW}, \mathrm{E}_{\mathrm{e} \max }=400 \mathrm{MeV}, \mathrm{Q}=350 \mathrm{pC}$ (b) $\mathrm{P}_{\text {las }}=200 \mathrm{TW}, \mathrm{E}_{\mathrm{e} \max }=800 \mathrm{MeV}, \mathrm{Q}=550 \mathrm{pC}$, (c) $\mathrm{P}_{\text {las }}=2 \mathrm{PW}, \mathrm{E}_{\mathrm{e} \max }=9 \mathrm{GeV}, \mathrm{Q}=1.8 \mathrm{nC}$ and (d) $\mathrm{P}_{\text {las }}=10 \mathrm{PW}, \mathrm{E}_{\mathrm{e} \max }=15 \mathrm{GeV}, \mathrm{Q}=4 \mathrm{nC}$ (Q being the electron beam charge and $\mathrm{E}_{\mathrm{e} \max }$ the maximum energy reached by the accelerated electrons). The computed $\beta$ tron spectra are shown on figure 7 (left). In addition to the overall increase of the radiated energy (pushed up by the charge), a shift of the $\beta$ tron spectrum toward higher energies is induced. Matching to the neon K-shell threshold is then improved and, even in the worst conditions (case (a)), a significant increase of the local gain coefficient (figure 7, right) is observed. The question is then: are these very high values observable?

\section{RADIATIVE TRANSFER MODELING}

A radiative transfer model has finally been coupled to the atomic kinetics code (introducing a new parameter: the length of the lasing medium $\mathrm{z}$ ) to evaluate the $\mathrm{X}$-ray laser output intensity ( $\mathrm{I}_{\mathrm{XRL}}$ ) and the incident laser intensity required to reach saturation (i.e. to reach an intensity $\mathrm{I}_{\text {sat }}$ above which amplification is no longer exponential: $\sim 810^{13} \mathrm{~W} / \mathrm{cm}^{2}$ in nitrogen) and produce a 'real' x-ray laser (XRL). The spatial evolution of the peak nitrogen $\mathrm{x}$-ray laser intensity is given on figure 8 for three different $\beta$ tron pump conditions: the experimental one (section 3 ) and two optimised cases (section 4 cases (b) and (c)). The pump duration has been set to $10 \mathrm{fs}$ and the density to $510^{20} / \mathrm{cm}^{3}$. No significant amplification can in fact be achieved (figure 8, left) as the x-ray laser output intensity stays far below 

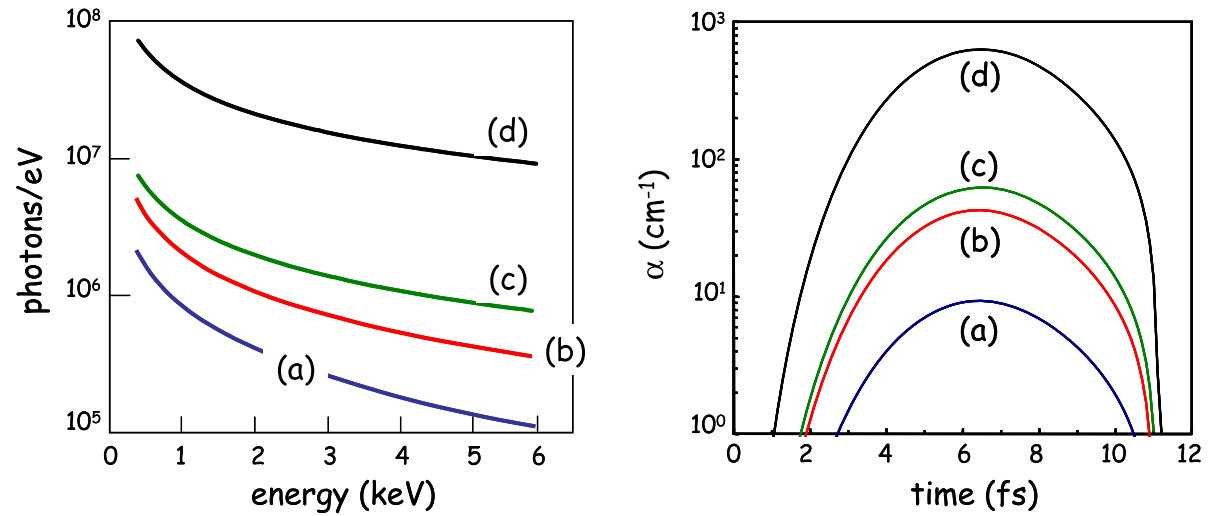

Figure 7. Optimised $\beta$ tron spectra (left) for the 4 investigated cases (as described in the text, the incident laser intensity and accelerated electron cut-off energy increasing from case (a) to case (d)) and corresponding local gain coefficients (right) in neon at $1.5 \mathrm{~nm}$, assuming a $10 \mathrm{fs}$ source duration and a density of $510^{20} / \mathrm{cm}^{3}$.
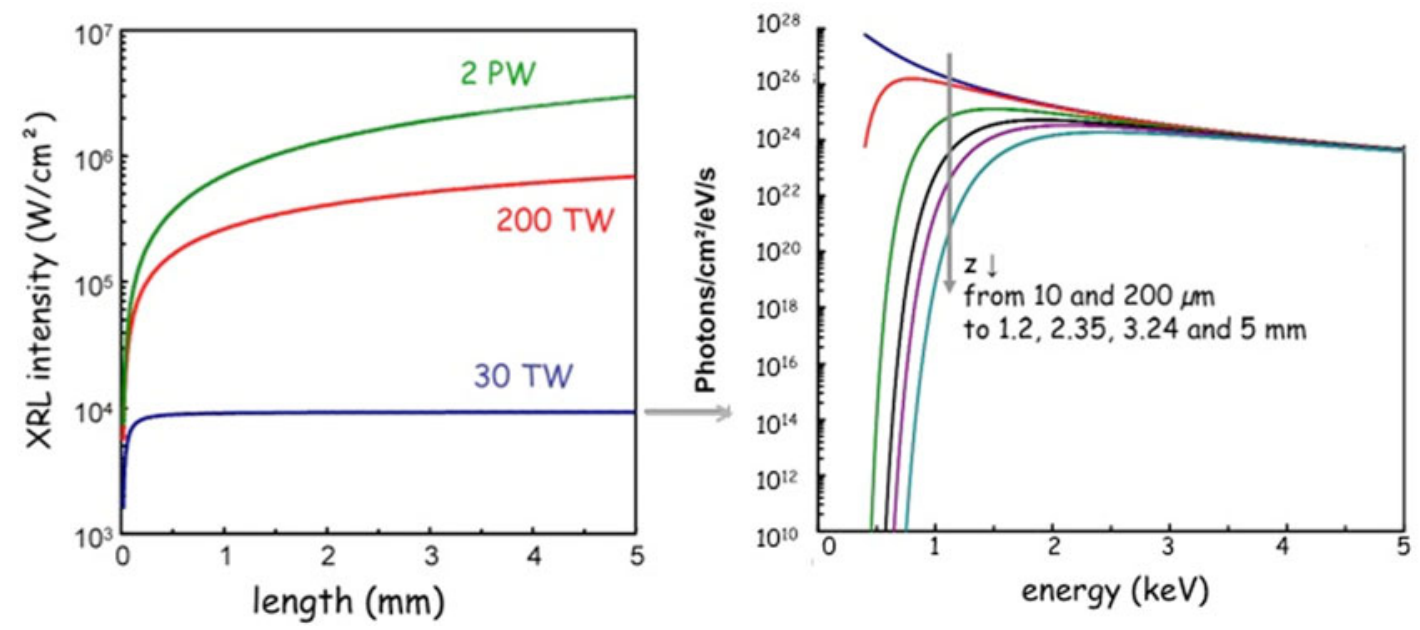

Figure 8. (left) Nitrogen x-ray laser output intensity as a function of the gas medium length for different pump conditions (assuming a $10 \mathrm{fs}$ duration and a $510^{20} / \mathrm{cm}^{3}$ initial density): 30 TW refers to the LOA experimental $\beta$ tron parameters, $200 \mathrm{TW}$ and $2 \mathrm{PW}$ to optimised calculations. (right) Evolution of the experimental $\beta$ tron spectrum with respect to the medium length.

the saturation intensity. Such a behaviour can be attributed to the fact that the pump is strongly absorbed by the lasing medium (figure 8 , right) and then, after a few hundreds of microns, is not brilliant enough to efficiently induce population inversion, the local gain coefficient decreasing from $65 \mathrm{~cm}^{-1}$ for $\mathrm{z}=10 \mu \mathrm{m}$ to $0.05 \mathrm{~cm}^{-1}$ for $\mathrm{z}=1.2 \mathrm{~mm}$. Lowering the medium density could of course improve the pump propagation but, as discussed in section 3 , the local gain coefficient will thus linearly decrease. Preliminary experiments in neon at 10 bars (figure 3 ) have been conducted at LOA and CEA/SLIC. Absorption of the $\beta$ tron radiation at $870 \mathrm{eV}$ (i.e. at the photo-ionisation threshold) has been clearly observed but no lasing effect along the $\mathrm{K} \alpha$ line detected, the pump intensity being far too low due to degraded $\beta$ tron divergence $(\sim 50 \mathrm{mrad})$, and thus poor focusing by the implemented toroidal mirror (focal spot radius $\sim 40 \mu \mathrm{m}$ ). 


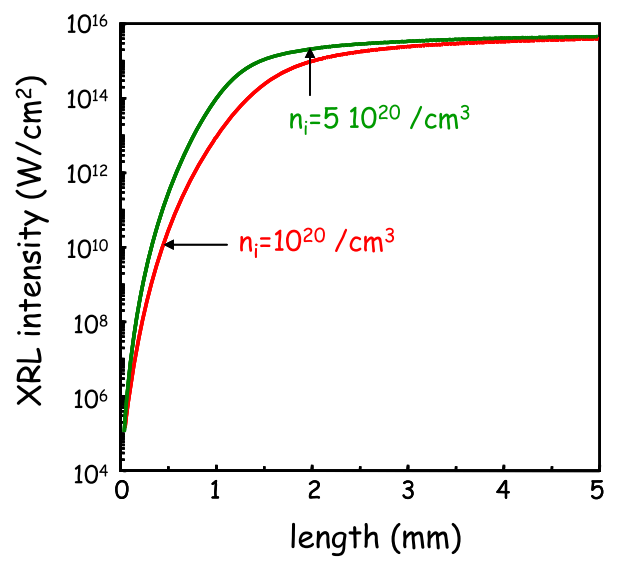

Figure 9. Nitrogen $\mathrm{x}$-ray laser output intensity as a function of the gas medium length (case (d), with a $10 \mathrm{fs}$ duration and an initial density of 1 or $510^{20} \mathrm{~cm}^{-3}$ ).
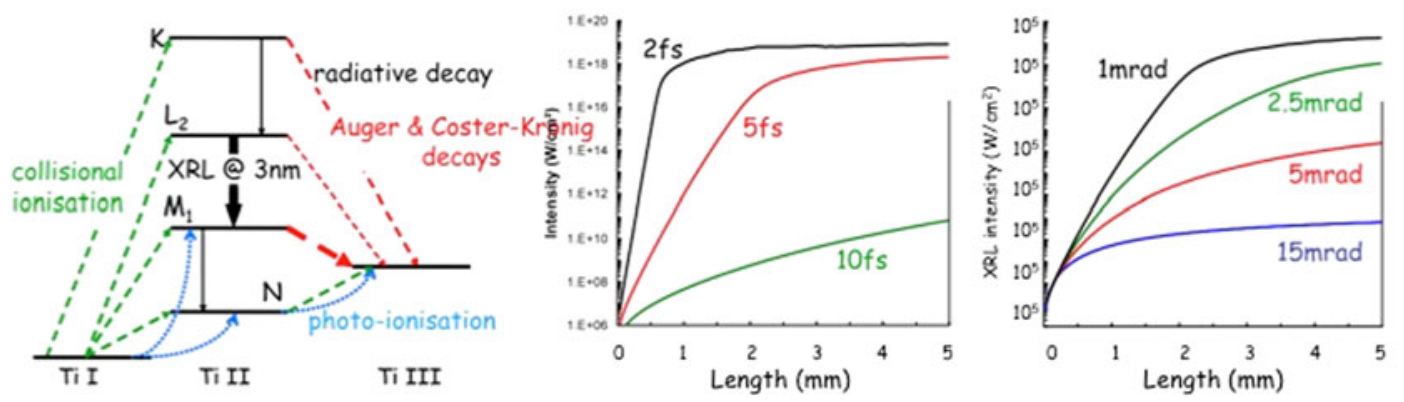

Figure 10. Partial energy diagram of a titanium collisional ionisation $x$-ray laser (left) and $x$-ray laser output intensity as a function of the medium length for (middle) various pump durations - at fixed divergence (1 mrad) - and (right) divergence - at fixed duration ( $2 \mathrm{fs}$ ). The computational parameters for the electron pump bunch are, respectively, $800 \mathrm{MeV} / 550 \mathrm{pC}[7]$ and $200 \mathrm{MeV} / 20 \mathrm{pC}$ [11].

For a density of $510^{20} \mathrm{~cm}^{3}$, it has been estimated that, at least, $710^{10}$ photons between 0.4 and $1.5 \mathrm{keV}$ (which corresponds to a $\beta$ tron pump intensity above $10^{16} \mathrm{~W} / \mathrm{cm}^{2}$ and roughly to the case (d) described previously) are needed to reach saturation in nitrogen (as shown on figure 9).

\section{CONCLUSION}

A coherent set of numerical codes has been developed to fully model the ISPI x-ray laser scheme, from pump propagation to atomic kinetics and radiative transfer. The study that has been conducted on a $\beta$ tron-pumped XRL indicates that lasing is strongly limited by the required pump energy and then that improvements are mandatory to decrease the $\beta$ tron divergence and boost its photon number: implementation of guiding techniques to increase the electron acceleration length [9], access to the next generation of multi-PW laser facilities. An alternative solution to more rapidly demonstrate this very promising scheme could be using a very powerful free electron laser, as investigated by $\mathrm{N}$. Rohringer et al. on the Stanford LCLS [10]. For a better coupling efficiency between the accelerated electron beam and the lasing medium, directly using this former could be mentioned, then basing the population inversion generation on collisional ionisation (and no longer photo-ionisation). In that case, the lasing material should have an intermediate atomic number (as titanium) to present a $\mathrm{N}$ shell, which allows 
depopulation of the lower lasing state by fast Auger and Coster-Kronig decays $(\sim 0.2 \mathrm{fs}-$ figure 10 , left). Preliminary calculations show that the accelerated electron bunch should be energetic enough (a few hundreds of MeVs) to limit scattering in the solid target (and keep beam collimation below a few mrads) and short enough (less than $5 \mathrm{fs}$ ) to compensate Auger and Coster-Kronig decays of the upper lasing state $(\sim 1.4 \mathrm{fs}$ ) (figure 10 ). This would be possible on multi-100TW laser facilities using colliding pulse injection, as developed at LOA by V. Malka and co-workers.

\section{Acknowledgment}

This work was supported by the Agence Nationale pour la Recherche through the COKER ANR-06-BLAN-012301 contract.

\section{References}

[1] Duguay M.A. and Rentzepis P.M., Appl. Phys. Lett. 10 (1967) 350.

[2] Kapteyn H.C., Appl. Opt. 31 (1992) 4931; Healy S.B. and Pert G.J., Inst. Phys. Conf. Ser. 151 (1996) 169; Moon S.J. and Eder D.C., Phys. Rev. A 57 (1998) 1391; Liu J. et al., Phys. Rev. A 63 (2001) 033809.

[3] Li Y. et al., Opt. Commun. 144 (1997) 118.

[4] Jacquemot S. et al., Springer Proc. Phys. 115 (2007) 321.

[5] Ribière M. et al., Appl. Phys. B 101 (2010) 753.

[6] Ta Phuoc K. et al., Phys. Rev. Lett. 97 (2006) 225002 \& Phys. Plasmas 14 (2007) 080701; Albert F. et al., Phys. Rev. E 77 (2008) 056402.

[7] Kneip S. et al., Phys. Rev. Lett. 103 (2009) 035002.

[8] Lu W. et al., Phys. Rev. STAB 10 (2007) 061301.

[9] Ribière M. et al., Applied Phys. B submitted.

[10] Rohringer N. and London R., Phys. Rev. A 80 (2009) 013809.

[11] Lund O. et al., unpublished. 